\title{
Accurate Quantification of Saccharin Using Isotope Dilution Liquid Chromatography Mass Spectrometry (ID-LC/MS)
}

\author{
Yunjung Lee ${ }^{\mathrm{a}, \mathrm{b}}$, Byungjoo Kim ${ }^{\mathrm{a}}$, Jeongkwon Kim ${ }^{\mathrm{b}}$, and Seonghee Ahn ${ }^{\mathrm{a}, *}$ \\ ${ }^{a}$ Division of Metrology for Quality Life, Korea Research Institute of Standards and Science, Yuseong, Daejon 305-600, Korea \\ ${ }^{b}$ Departments of Chemistry, Chungnam National University, Daejeon, Korea
}

Received May 11, 2011; Revised June 3, 2011; Accepted June 8, 2011

First published on the web June 15, 2011; DOI: 10.5478/MSL.2011.2.2.037

\begin{abstract}
Saccharin is a commonly used artificial sweetener in foodstuffs. However, for its carcinogenic dispute, it has been regulated by government bodies. In this study, isotope dilution mass spectrometry (ID-MS) was introduced for the accurate quantification of saccharin. To employ ID-LC/MS, we obtained its isotope analogue, ${ }^{13} \mathrm{C}_{1}$-sodium saccharin, by customized synthesis. Samples were spiked with ${ }^{13} \mathrm{C}_{1}$-sodium saccharin and analyzed with LC/MS in negative mode. Chromatographic conditions were optimized for the adequate chromatographic retention and separation of saccharin with a $\mathrm{C}_{18}$ column. MS was operated with electrospray ionization by the selected ion monitoring (SIM) mode of $[\mathrm{M}-\mathrm{H}]^{-}$for saccharin $(\mathrm{m} / \mathrm{z} 182)$ and $[\mathrm{M}-\mathrm{Na}]^{-}$for its isotope analogue $(\mathrm{m} / \mathrm{z} 183)$. To validate the ID-LC/MS method for accurate measurement, we prepared a batch of a candidate material by sortifying quasi-tea-drinks with saccharin and analyzed samples gravimetrically fortified in various levels of concentration. The repeatability and reproducibility of this method was tested by analyzing the reference material. Result show that ID-LC/MS is a reliable method for the quantitative analysis of saccharin.
\end{abstract}

Key words: Saccharin, Isotope analogue- ${ }^{13} \mathrm{C}_{1}$-sodium saccharin, Isotope dilution mass spectrometry (ID-MS), Repeatability, Reproducibility

\section{Introduction}

Saccharin is one of the popular non-caloric artificial sweeteners. ${ }^{1}$ The potency of pure saccharin as a sweetener is about 700 times that of sugar. ${ }^{2}$ It is very important to diabetic patients to control their insulin and body weights as a substitute for sugar. However, the dispute concerning its safety for human consumption has been issued continuously since saccharin has been synthesized in $1879 .{ }^{1,3}$ Some reports for toxicological tests with saccharin have shown that it caused bladder tumors, renal effects and digestive disturbances in saccharin treated male rats, while opponents have insisted that they occurred inconsistently and with high does. ${ }^{3,4}$ These days saccharin has been allowed to use in some foods, but still its maximum contents is regulated. In Korea, the use of saccharin is allowed only in some foodstuffs such as Kimchi, drinks, and patient's food and acceptable daily intake is set to $0 \sim 5 \mathrm{mg} / \mathrm{kg}$ body weight.

The analysis of saccharin has been accomplished with various chromatographic methods such as thin layer chromatography, ${ }^{5}$ high performance liquid chromatography (HPLC), ${ }^{6-9}$ ion chromatography, ${ }^{10}$ gas chromatography ${ }^{11-12}$ and capillary electrophoresis ${ }^{13-14}$ with several detection

*Reprint requests to Dr. Seonghee Ahn

E-mail: sahn@kriss.re.kr techniques. Only a few LC/MS methods have been performed for the determination of saccharin up to date. Koyama, et. al. ${ }^{15}$ and Yang et. al. ${ }^{16}$ have developed an LC/MS method with an ion-paring reagent for better separation from other sweeteners. However, ion-paring reagents such as dibutylammonium acetate and triethylamine might suppress the ionization of saccharin in electrospray processes. Ferrer et. al. ${ }^{17}$ has reported the quantitative analysis of saccharin and other sweeteners in surface water, ground water and wastes water samples with LC/TOF-MS by matrix-matched standard calibration.

In this study, a method for the accurate quantification of saccharin was established using ID-LC/MS with higher-order metrological quality. The ID-LC/MS method has been known to provide reliable and reproducible results for the accurate determination of saccharin without systematic bias. However, an isotope analogue of saccharin is not commercially available, thus we obtained its isotope analogue by customized synthesis. Therefore, it is the first time to employ ID-LC/MS for saccharin analysis. LC separation was achieved by a C18 column with optimized mobile phase conditions for the stable retention and separation of saccharin. For the validation of this method, we prepared reference materials by fortifying quasi-tea-drinks with saccharin. The repeatability and reproducibility of the method were examined with the reference materials. 


\section{Experimental}

\section{Materials}

Saccharin was obtained from LGC Promochem (Middlesex, $\mathrm{UK})$ and its purity was $(99.6 \pm 0.6) \%$ in mass fraction by a manufacturer. Its corresponding isotope, ${ }^{13} \mathrm{C}_{1}$-sodium saccharin was a custom-ordered product by Chemizon (Sungnam, Korea). Purity of ${ }^{13} \mathrm{C}_{1}$-sodium saccharin was $89.9 \%$ (at $254 \mathrm{~nm}$ ) by a manufacturer. HPLC grade acetonitrile was purchased from Burdick and Jackson (Muskegon, MI, USA) and ammonium acetate and acetic acid were from Aldrich (Milwaukee, WI, USA).

\section{Standard solution}

Standard solutions of saccharin were prepared and verified in our laboratory. The procedure for preparation and verification was published elsewhere. ${ }^{18-19}$ Briefly, four replicates of stock solutions were gravimetrically prepared in $50 \mathrm{mmol} / \mathrm{L}$ ammonium acetate buffer ( $\mathrm{pH} \mathrm{4.5)}$ at the level of $100 \mathrm{mg} / \mathrm{kg}$. $\mathrm{A}^{13} \mathrm{C}_{1}$-sodium saccharin solution was prepared in the same way. Two isotope ratio standard solutions for each of four stock solutions were prepared by gravimetrically mixing with isotope solution as 1:1 isotope ratio. Based on the results of LC/MS, consistency among eight isotope ratio standards was evaluated and then, one isotope ratio standard was selected and used for calibration and further sample analysis.

\section{Preparation of samples for validation}

For the validation of our method, we prepared samples with known levels of saccharin by gravimetrically fortifying blank quasi-tea-drinks in the range of $50-1,000 \mathrm{mg} / \mathrm{kg}$. Quasitea-drinks were obtained from a local market and whose saccharin level was tested to be below the detection limit of this method. To test the repeatability and reproducibility of the method, we prepared a batch of a candidate reference material for saccharin analysis as following in parallel with this study. Quasi-tea-drinks were collected in one big bottle, after then the drink sample was fortified with concentrated saccharin solution. This bulk solution was homogenized for 5 hours under argon gas and bottled into a $200 \mathrm{~mL}$-polyethylene jar. The sealed bottles were stored at 4 before analysis.

\section{LC/MS analysis}

For the ID-LC/MS analysis of the sample, $1 \mathrm{~g}$ of sub-sample was spiked with an appropriate amount of the ${ }^{13} \mathrm{C}_{1}$-sodium saccharin solution to make $1: 1$ isotope ratio and then filtered by using a nylon membrane filter (Waters Corp. Clifton, NJ, USA). The LC/MS analysis of saccharin was performed with a Thermo TSQ Quantum mass spectrometry (San Jose, CA, USA) which is interfaced with an Agillent Technologies 1100 series LC system (Palo Alto, CA, USA). The ESI probe voltage was $-3,000 \mathrm{~V}$ and skimmer offset voltage was $16 \mathrm{~V}$. The gas flow rate for nebulization and desolvation was operated with $\mathrm{N}_{2}$ gas and the capillary voltage for desolvation was $270{ }^{\circ} \mathrm{C}$. An LC column was a Prodigy ODS-3 column (C18,
$5 \mu \mathrm{m}, 4.6 \times 250 \mathrm{~mm}$, Phenomenex Co., Torrance, CA, USA) at a flow rate of $1.0 \mathrm{~mL} / \mathrm{min}$ with a guard column of the same phase. Mobile phase consisted of $50 \mathrm{mmol} / \mathrm{L}$ ammonium acetate as a solvent $\mathrm{A}$ and acetonitrile as a solvent $\mathrm{B}$. The separation of saccharin was achieved by isocratic elution with $10 \% \mathrm{~B}$ for $12 \mathrm{~min}$, followed by changing to $100 \% \mathrm{~B}$ for $4 \mathrm{~min}$ to wash the column and to initial phase for another $3 \mathrm{~min}$ for the next run. For quantitative analysis, the mass spectrometer was operated in negative mode and selected ion monitoring (SIM) mode; [M-H] for saccharin $(\mathrm{m} / \mathrm{z} 182)$ and $[\mathrm{M}-\mathrm{Na}]^{-}$for its isotope analogue $(\mathrm{m} / z \mathrm{183})$. The main peak $(\mathrm{m} / \mathrm{z} 183)$ of ${ }^{13} \mathrm{C}_{1}$-sodium saccharin is overlapped with the natural isotope of saccharin, thus the natural isotope ratio of saccharin was corrected in calculation.

\section{Calculations}

The mathematical model for the IDMS measurements of an analyte by one point calibration (with isotope-ratio matching) can be written as

$$
\begin{aligned}
& C_{\text {sample }}=\frac{M_{i s-\text { sol,spiked }} \cdot A R_{\text {sample }} \cdot M_{s-\text { sol,std.mix }} \cdot C_{s-\text { sol }}}{M_{\text {sample }} \cdot A R_{\text {std.mix }} \cdot M_{i s-s o l, \text { std.mix }}}(1) \\
& \text { where, } \\
& C_{\text {sample }} \quad \text { is the concentration of saccharin in the sample; } \\
& C_{\text {s-sol }} \text { is the concentration of the calibration standard } \\
& \text { solution; } \\
& M_{\text {sample }} \quad \text { is the mass of the sample taken for analysis; } \\
& M_{\text {is-sol, spiked }} \text { is the mass of the }{ }^{13} \mathrm{C}_{1} \text {-sodium saccharin standard } \\
& \text { solution added to the sample aliquot; } \\
& M_{\text {is-sol, std. mix. }} \text { is the mass of the }{ }^{13} \mathrm{C}_{1} \text {-sodium saccharin standard }
\end{aligned}
$$

\section{Results and Discussions}

\section{Optimization for ID-LC/MS method}

Figure 1 represents chemical structures of saccharin and ${ }^{13} \mathrm{C}_{1}$-sodium saccharin. We carefully examined their mass<smiles>O=C1NS(=O)(=O)c2ccccc21</smiles>

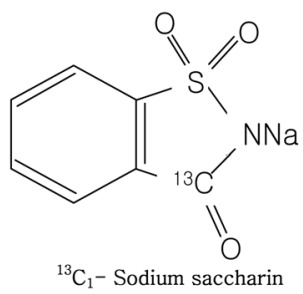

Figure 1. Structures of saccharin and ${ }^{13} \mathrm{C}_{1}$-sodium saccharin. 
spectra at the level of $1 \mathrm{mg} / \mathrm{kg}$ for saccharin and $2 \mathrm{mg} / \mathrm{kg}$ for ${ }^{13} \mathrm{C}_{1}$-sodium saccharin. As shown in Figure 1, there are no serious impurity peaks in mass spectrum of ${ }^{13} \mathrm{C}_{1}$-sodium saccharin. Saccharin was detected as a deprotonated ion $[\mathrm{M}-\mathrm{H}]^{-}$at $\mathrm{m} / \mathrm{z} 182$ and ${ }^{13} \mathrm{C}_{1}$-sodium saccharin was detected as a desodiated ion [M-Na] $]^{-}$at $\mathrm{m} / \mathrm{z} 183$ in negative mode.

Conditions of chromatographic separation were optimized without ion-pairing reagents. For the stable retention and the proper sensitivity of saccharin, the isocratical flow of $90 \%$ mobile phase A (ammonium acetate buffer) and 10\% mobile phase B were used with a C18 column. Typical LC/MS chromatograms of saccharin $(100 \mathrm{mg} / \mathrm{kg})$ and ${ }^{13} \mathrm{C}_{1}$-sodium saccharin $(50 \mathrm{mg} / \mathrm{kg})$ in isotope ratio standard (a) and in drink sample (b) by the SIM mode are shown in Figure 3. Blank test of drink sample at m/z 182 and 183 was performed, but there was no serious signal from drink sample. Saccharin and ${ }^{13} \mathrm{C}_{1}$-sodium saccharin were eluted at $8.5 \mathrm{~min}$. The method using a selected reaction monitoring (SRM) mode was performed; however, the sensitivity of fragment peaks was not enough for quantitative analysis. Therefore, the SIM mode was used

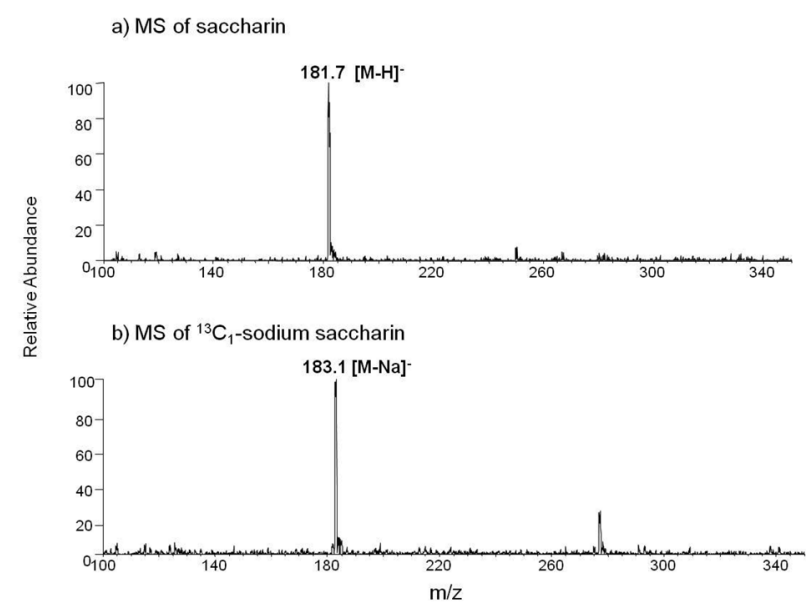

Figure 2. MS spectra of saccharin (a) and ${ }^{13} \mathrm{C}_{1}$-sodium saccharin (b) obtained in negative mode.

a) Standard solution

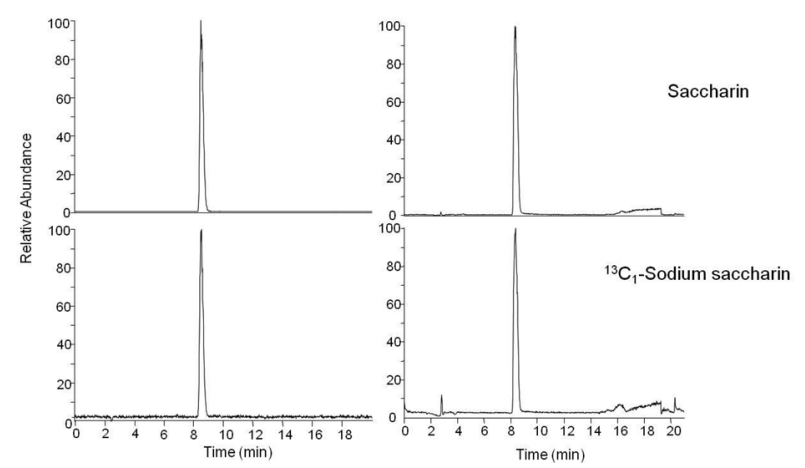

Figure 3. LC/MS chromatograms of saccharin $(100 \mathrm{mg} / \mathrm{kg})$ and ${ }^{13} \mathrm{C}_{1}$-sodium saccharin (50 mg/kg) in isotope standard ratio (a) and fortified quasi-tea-drink (b) by single ion monitoring mode (SIM). for the quantitative analysis of saccharin using the ID-LC/MS method. The detection limit of the LC/MS method for saccharin was $0.05 \mathrm{mg} / \mathrm{kg}$ with signal to ratio of 3 , which was estimated based on the signal to ratio of the SIM chromatograms.

\section{Validation of ID-LC/MS}

To validate the analytical method, gravimetrically fortified samples at various levels of concentration, in the range of $50 \sim 1,000 \mathrm{mg} / \mathrm{kg}$, were prepared. Table 1 is the LC/MS results of saccharin in those fortified samples. The concentrations measured by LC/MS agree well with the fortified concentrations within their uncertainties.

In addition, another way to validate the analytical method is the use of certified reference materials (CRMs) or highly homogenized reference materials. However, there are no CRMs for saccharin in drink, thus we decided to prepare a candidate reference material in our laboratory by fortifying quasi-teadrink with saccharin to use it as a homogeneous sample for a repeatability and a reproducibility test. Our laboratory had experiences to produce and distribute several classes of the certified/reference materials as the national metrology institute of Korea. The procedure of preparation for the reference material for drink sample is described elsewhere. ${ }^{18-19}$

Ten bottles were selected from the batch and one sub-sampling from each bottle was analyzed for certifying the reference material in along with the repeatability test by the ID-LC/MS method. The results are listed in Table 2. The reference value was $290.2 \pm 9.6 \mathrm{mg} / \mathrm{kg}$ with the expanded uncertainty in $95 \%$ confidence level $(k=2.12)$. Details about the estimation of the reference value and expanded uncertainty are described in an article $^{20}$ from our laboratory, thus not described here. The relative standard deviation among ten bottles was $1.4 \%$, which is assumed to be mostly contributed by the repeatability of the measurement method as among-bottle inhomogeneity for liquid sample is usually negligible. The reproducibility test was carried out with the same method after two months later by analyzing five bottles from the batch as a part of stability test of the material. The value was $295.0 \pm 18.3 \mathrm{mg} / \mathrm{kg}$, which agree within the initial reference value, indicating

Table 1. ID-LC/MS results of gravimetrically fortified samples at the various levels of saccharin in quasi-tea-drink.

\begin{tabular}{ccc}
\hline \hline $\begin{array}{c}\text { Fortified concentraion } \\
(\mathrm{mg} / \mathrm{kg})\end{array}$ & $\begin{array}{c}\text { Measured concentraion } \\
(\mathrm{mg} / \mathrm{kg})\end{array}$ & Ratio (\%) \\
\hline 48.50 & $48.96 \pm 0.19^{\mathrm{b}}$ & 100.9 \\
106.63 & $106.38 \pm 0.52$ & 99.8 \\
320.46 & $320.21 \pm 1.51$ & 99.9 \\
984.83 & $983.64 \pm 3.91$ & 99.9 \\
\hline
\end{tabular}

${ }^{\mathrm{a}}$ The uncertainty of the gravimetrically fortified level is rather minimal compared to the uncertainty in analytical measurement, thus is not reported in this table.

${ }^{\mathrm{b}}$ The number following " \pm " is the expanded uncertainty with a level of confidence of $95 \%$. 
Table 2. Results of saccharin in quasi-tea drink by ID-LC/MS in two different time periods within 2 months.

\begin{tabular}{ccc}
\hline \hline & \multicolumn{2}{c}{ Results of ID-LC/MS (mg/kg) } \\
\hline & Sub-sample \#1-1 & 291.7 \\
Sub-sample \#1-2 & 280.9 \\
Sub-sample \#1-3 & 294.3 \\
& Sub-sample \#1-4 & 291.2 \\
& Sub-sample \#1-5 & 293.6 \\
Sub-sample \#1-6 & 288.7 \\
Period 1 & Sub-sample \#1-7 & 288.1 \\
& Sub-sample \#1-8 & 289.7 \\
& Sub-sample \#1-9 & 288.1 \\
& Sub-sample \#1-10 & 295.0 \\
\cline { 2 - 3 } & Average & 290.2 \\
& RSTD & $1.4 \%$ \\
& Expanded uncertainty & 9.6 \\
\hline \multirow{2}{*}{ Period 2 } & Sub-sample \#2-1 & 285.4 \\
& Sub-sample \#2-2 & 297.3 \\
& Sub-sample \#2-3 & 298.5 \\
& Sub-sample \#2-4 & 296.0 \\
& Sub-sample \#2-5 & 297.5 \\
\hline & Average & 295.0 \\
& RSTD & $1.8 \%$ \\
& Expanded uncertainty & 18.3 \\
\hline
\end{tabular}

${ }^{\mathrm{a}}$ The expanded uncertainties are with a level of confidence of $95 \%$.

that the proposed method provided good reproducibility. The experiments with fortified samples at various levels of saccharin and the reference materials proved that the proposed method is reliable to determine the accurate amount of saccharin in drinks.

\section{Conclusions}

An approach for the simple and accurate analysis of saccharin was performed using the customized isotope of saccharin by the ID-LC/MS method. The results of ID-LC/MS with gravimetrically fortified samples at the various levels showed that the proposed method is appropriate for the analysis of saccharin in drink. For the validation of our method, the fortified quasi-tea-drink reference materials were prepared and evaluated in our laboratory. Repeatability was performed with ten bottles and the relative standard deviation among reference materials was $1.4 \%$. The reference value of quasitea-drink reference materials for saccharin analysis was $290.2 \pm 9.6 \mathrm{mg} / \mathrm{kg}$ and the result of the reproducibility test was $295.0 \pm 18.3 \mathrm{mg} / \mathrm{kg}$ after two months later. The repeatability and reproducibility test proved that the ID-LC/MS method for saccharin analysis has high metrological quality as a reference method.

\section{References}

1. Remsen, I.; Fahlberg, C. Am. Chem. J. 1879, 1, 426.

2. Arnold, D. L.; Krewski, D.; Munro, I. C. Toxicology 1983, $27,179$.

3. Oser, B. L. Fd. Chem. Toxic. 1985, 23, 535.

4. Bryan, G T.; Erturk, E.; Yoshida, O. Science 1970, 168, 1238.

5. Holder, C.L.; Bowman, M.C. Toxicol. Lett. 1980, 5, 27.

6. Galli, V.; Barbas, C. J. Chromatogr. A 2004, 1048, 207.

7. Wasik, A.; McCourt, J.; Buchgraber, M. J. Chromatogr. A 2007, 1157, 187.

8. Di Pietra, A. M.; Cavrini, V.; Bonazzi, D.; Benfenati, L. Chromatographia 1990, 30, 215.

9. Tyler, T. A. J. Assoc. Off. Anal. Chem. 1984, 67, 745.

10. Zhu, Y.; Guo, Y.; Ye, M.; James, F. S. J. Chromatogr. A 2005, 1085, 143.

11. Lho, D. S.; Hong, J. K.; Paek, H. K.; Lee, J. A. J. Anal. Toxicol. 1990, 14, 77.

12. Heitkemper, D. T.; Jackson, D. S.; Kaine, L. A.; Mulligan, K. A.; Wolnik, K. A. J. Chromatogr. A 1994, 671, 323.

13. Thompson, C. O.; Trenerry, V. C.; Kemmery, B. J. Chromatogr. A 1995, 704, 203.

14. Boyce, M. C. J. Chromatogr. A 1999, 847, 369.

15. Koyama, M.; Yoshida, K.; Uchibori, N.; Wada, I.; Akiyama, K.; Sasaki, T. J. Food Hyg. Soc. Japan 2005, 46, 72.

16. Yang, D.-J.; Chen, B. J. Agric. Food Chem. 2009, 57, 3022.

17. Ferrer, I.; Thyrman, E. M. J. Chromatogr. A 2010, 1217, 4127.

18. Lee, I.; Ahn, S.; Kim, B.; Hwang, E.; Kim, Y.-S. Bull. Korean Chem. Soc. 2008, 29, 2125.

19. Park, S.; Kim, B.; So, H.-Y.; Kim, Y.-J.; Kim, J. Bull. Korean Chem. Soc. 2007, 28, 737.

20. Kim, B.; Park, S.; Lee, I.; Lim, Y.; Hwang, E.; So, H.-Y. Anal. Bioanal. Chem. 2010, 398, 1035. 